\title{
Regular and analogue black holes: Parallel session report
}

\author{
Carlos Barceló \\ Instituto de Astrofísica de Andalucía (IAA-CSIC) \\ Glorieta de la Astronomía, 18008 Granada, Spain \\ E-mail: carlos@iaa.es
}

Stefano Liberati

International School for Advanced Studies

Via Bonomea 265, I-34136 Trieste, Italy

and INFN, Sezione di Trieste, Trieste, Italy

E-mail: liberati@sissa.it

\begin{abstract}
This is a rapporteur article of the parallel session "Regular and analogue black holes" which took place within the 14th Marcel Grossmann Meeting.
\end{abstract}

\section{Introduction}

Black holes have been and still are central to the development of General Relativity. They are not only the most pure and paradigmatic gravitational objets but they have also led us to the incorporation of quantum phenomena in gravitational settings. As it often happens with important discoveries, the realisation by Hawking that black holes can evaporate by quantum effects led to new pressing questions which in turn have led to a very fruitful stream of research. Among these questions a special status can be ascribed to the so-called information loss and transplanckian problems. Precisely under the pressing need to address such issues, the last decades have seen the momentous development of two lines of research, namely, regular black holes - for what regards the information problem - and analogue black holes - for what regards the transplanckian problem.

This parallel session tried to offer a panoramic view (alas incomplete) of these research areas. This report intends to offer guidance about the different themes touched within the parallel session, while leaving the details of the presented works to the proceedings presented by the speaker themselves.

\section{Regular Black Holes}

Most GR practioners believe that classical black holes will be subjected to some form of regularisation by quantum gravity effects which will end up eliminating their classical singularities. There are however many different proposals regarding what sort of physics might be underneath this regularisation. For example, Sakharov Ref. 1 and Gliner Ref. 2 already in the sixties suggested that at the high densities attained close to singularity formation, the effective matter content might develop a vacuum-like equation of state, $\rho=-p$, providing a repulsive force. On the other hand, soon after, Bardeen showed that it was possible to have black hole geometries 
(here meaning having an event horizon) satisfying the null energy condition (the one relevant to Penrose's singularity theorem Ref. 3) but having no singularities (see e.g. Ref. 4 and Ref. 5). Singularity (and singularity theorem) avoidance was caused by the non-existence of an open Cauchy surface: the spacetime develops a topol-

ogy change from open hypersurfaces to closed ones. ${ }^{6}$ Matter crossing the black-hole horizon and falling towards the apparent singularity would end up reappearing from a white-hole horizon into a different universe, not in the same asymptotic region. Bardeen's spacetime and others with similar structure (e.g. Ref. 7) are examples of how one can regularise black hole singularities without affecting their event horizon.

Hawking's idea that event horizons should evaporate, leading eventually to some fundamental lost of information, introduced a new facet to the singularity problem. The presence of the singularity implies the incompleteness of the future null infinity which, together with the hypothesis of complete thermal evaporation of the black hole, entails the impossibility of recovering in the future all the information present on the past null infinity.

After the information problem was posed, many different evaporation scenarios have been put forward to resolve it via an effective removal of the singularities associated with black holes (see e.g. Ref. 8). While the parallel session could not provide a complete overview of all these approaches, we will describe the different specific contributions made to this meeting by presenting a brief summary of each contribution following the presentation order.

\section{1. "Quantum Black Holes and Unitarity" by Abhay Ashtekar}

The first talk of the session was given by Abhay Ashtekar. After a clear cut review of the information loss paradox, he passed on to describe what he thinks as the two crucial ingredients in solving the problem. The first ingredient is the possibility of having a singularity resolution. It was indeed stressed that, for example, within the firewall proposal there is as hidden assumption that the singularity is not resolved. He argues that with singularity resolution the firewall argument fails as in general a black hole can store a large amount of information within its (potentially very large) volume, which is not necessarily accounted for by the Bekenstein-Hawking entropy. The second ingredient is the notion of quasi-local horizon. Ashtekar explained in detail the crucial difference between event and quasi-local horizons, stressing the importance of distinguishing these notions in order to properly address the problem. Clarified these points, the presentation moved on focusing on a specific toy model for 2D black holes: the Callen-Giddings-Harvey-Strominger (CGHS) model. Ashtekar considered a refinement of the CGHS model which introduces back reaction treated via a mixture of analytical techniques and numerical simulations. It was shown that within this model the classical singularity is tamed by the backreaction: there is not thunderbolt singularity nor event horizon. In conclusion, the future geometry will be asymptotically flat and with a complete future null infinity. It was then claimed that this behaviour should persist in a full $4 \mathrm{D}$ theory and that once the event horizon 
is replaced by a local concept such as a trapping horizon, most of the paradoxes go away. Furthermore, an evaporating quasi-local horizon will be in general timelike so information can leek out without violation of causality. The final part of the talk was devoted to two possible scenarios for the recovery of the information, one of Page type - correlations are restored when the black hole is still macroscopic at the cost of giving up the generalised second law - and one of Ashtekar-Bojowald type - correlations are restored when the black hole is microscopic. The second mechanism would imply a restoration of unitarity though the emission of extremely infrared radiation over long times. This radiation would leek out from the quantum region replacing the singularity, but the presentation closed stressing that a more complete understanding of this mechanism and the quantum region is required.

\section{2. "Purifying Radiation from Regular Black Holes" by Eugenio Bianchi}

The following talk by Eugenio Bianchi was mainly dedicated to the possibility of retrieval of information in a regular black hole scenario, via the purification of the initially thermal radiation emitted by the evaporating black hole. After presenting the rationale behind regular black hole proposals, Bianchi stressed the necessity of understanding the details of the purification process to asses the viability of regular black holes as solutions of the information loss problem. In this respect, the presentation proceed by separately discussing the flux associated to Hawking radiation, its entanglement entropy and the relation between the two. The speaker presented calculations of the fluxes and entanglement entropies in three regular black hole scenarios showing that an appropriate purification process (à la Page) generically seems to entail the presence of negative energy fluxes. At the end of the talk some estimates of the duration of the adiabatic evaporation (standard Hawking phase) were given showing that this can last very long, on timescales comparable to the standard scenario.

\section{3. "A New Decay Mode for Black Holes" by Hal Haggard}

In his talk, Hal Haggard started reviewing some previous works describing possible regular evaporations of black holes, in particular the early works by FrolovVilkovisky (see Ref. 9, 10), Stephens-t' Hooft-Whiting (see Ref. 11) and AshtekarBojowald (see Ref. 13). He then argued that these works suggest to look for the possibility of completely time symmetric diagrams. This would involve pasting a black hole spacetime with a white hole spacetime. After mentioning as inspiring ideas the more recent works by t'Hooft (see Ref. 14) and Gambini-Pullin (see Ref. 15, 16) he passed on to describe a specific method for constructing such timesymmetric spacetimes. The method basically consisted in an appropriate "cut and paste" starting from two copies of the Kruskal manifold. He argued that this geometry can be justified as a quantum tunnelling between a collapsing phase and an 
expanding phase; the tunnelling or bouncing phase will correspond to a quantum region (in this context also the work by Hájíček-Kiefer Ref. 12 was mentioned as sharing a similar in spirit). The speaker reported that the time scale they obtain for the tunnelling to develop scales with $\sim M^{2}$ in contrast with the much larger Hawking evaporation time scale $\sim M^{3}$. He also reported, that this construction also implies that quantum effects cannot be confined to the interior of the black hole region but have to necessarily extend beyond the horizon. It was noticed that this is also the case for a similar black hole-white hole setting recently proposed by Barceló-Carballo-Garay-Jannes (Ref. 17, see also report on Garay's talk). After some discussion of a possible source of instability due to the presence of a white horizon, Haggard closed its seminary by suggesting that this results seem to corroborate the hypothesis that a black hole be a bouncing star seeing from the outside in slow motion.

\section{4. "Quantum Black Holes Without Singularity" by Claus Kiefer}

In this talk Claus Kiefer reviewed a model of singularity avoidance he and Piotr Hájíček worked out several years ago Ref. 12 which is now capturing a renewed attention because its relation with the proposals of Ref. 17, 18. The model consists on a reduced quantization of a spherically-symmetric null dust shell. The classical theory contains a collapsing solution towards a singularity and a solution expanding from a singularity. The quantum theory instead contains a superposition of these two branches, with no signs of a singularity. This is argued for because the complete wave function associated with an in-going wave packet in the past is zero at $r=0$, interpreted as meaning that the probability of finding the shell with a zero radius vanishes. Semiclassically, this would be interpreted as a regular transition between a black and a white hole solution, with no event horizon ever forming. Regarding the duration of the transition, within this formalism it is not easy to find a clear answer but further development hopefully will address this issue. On that regard we must point to the work of Ambrus and Hajicek. ${ }^{19}$

\section{5. "Do Stars Die Too Long?" by Luis J. Garay}

In his talk Luis J. Garay started by mentioning the problem of singularities in classical General Relativity and the information-loss problem when black holes are subject to quantum effects. To these problem he added the lifetime problem of evaporating black holes: an evaporating solar-mass black hole would enter into a proper quantum realm after $10^{67}$ years, orders of magnitude larger than the age of the universe. As such we face the problem to ever be able to tests Hawking's prediction in astrophysics. Garay argued, that the existence of this problem is among the motivations for considering viable alternatives to the standard process of gravitational collapse to forming black holes. The seminar then presented and discussed an alternative scenario based on an ultra-fast transition (as seen even 
from far away observers) between a black hole and a white hole spacetimes. The specific heuristic geometry discussed has as main characteristics: i) that there is a quantum non-standard region extending outside the gravitational radius, and ii) that the total duration of the bounce as seen by far-away observers is essentially twice the collapsing proper time, a few microsecond for neutron-like stars collapsing almost in free fall. While the first feature is shared by the similar model presented by Haggard, the second is peculiar of this proposal. The discussion followed up by focussing on the form of the transient period leading to the stationary regime. The scenario implies a serie of several collapses and bounces of the entire body, which opens up the possibility of finding new forms of equilibrium distinct from standard black holes. These could be black stars, objects hovering near but outside their gravitational radii. Garay argued that in principle one could experimentally distinguish a black star from a black hole. In any case he stressed that the physics of the transient phase and that of the final equilibrium object are in principle two independent issues which could and should be addressed separately.

\section{6. "Absorption of Planar Massless Scalar Waves by Bardeen Regular Black Holes" by Caio Filipe Bezerra Macedo}

Can a regular black holes be distinguished from standard ones by measuring their scattering cross sections? This is the question addressed by Caio Macedo in his talk. Specifically he considered Bardeen's regular black holes. ${ }^{4}$ Bardeen's metric for a given mass differs everywhere from its Schwarzschild counterpart, not just in a small region around the singularity. Therefore, this specific regular black holes could in principle be distinguished. He presented Area and Absortion-Cross-Section (ACS) values for Bardeen metrics with different charge parameters $q$. He reported that Bardeen's ACS are always smaller than Schwarzschild ACS. On the other hand, a Reissner-Nordström solution with equal charge than its Bardeen counterpart has a smaller ACS.

\section{7. "Isomonodromy, Painlevé and Black Hole Scattering" by Bruno Carneiro da Cuhna}

In the next talk Bruno Carneiro da Cuhna presented new mathematical tools to obtain scattering coefficients for black holes, with a special emphasis in scattering between different asymptotic regions. He presented results on when it is possible to analyse a scattering problem through a system of ordinary differential equations with some singular points (Fuchsian equations). He related this property to the particularity of the analysed black hole spacetimes of possessing a principal conformal Killing-Yano tensor. Then, the scattering amplitudes in different black spacetimes can be obtained through monodromy (one turn around) data around singular points of the ordinary differential equations. He stressed the utility that these techniques could have in studying CFT phenomena through the AdS/CFT correspondence. 


\section{8. "QFT on Curved Spacetime with Non Vacuum States: Implications for Hawking Radiation" by Kinjalk Lochan}

Hawking and Unruh effects result from realising that what for some observers is a vacuum state for others (relative accelerated observers) corresponds to a thermal distribution of particles. But what happens when starting not from a vacuum state but from a state with some particles present? This is the problem which was discussed in Kinjalk Lochan's talk. For instance, if the quantum in-state of a field in a black hole is not vacuum but contains one particle, this translates into a distortion from thermality in the out-state. These distortion appear predominantly in the IR part of the spectrum. It was argued that therefore the outgoing radiation do have information about the initial state of the matter that went to form the black hole, which although effectively classical is quantum in its essence.

\section{Analogue Black Holes}

The analogue gravity programme aims at gaining new insights into gravitational physics by looking at condensed matter models, suitable to be built in a laboratory, which can simulate emergent spacetimes and their phenomenology. These systems, such as e.g. acoustics in flowing fluids, light in moving dielectrics, or quasiparticles in a moving superfluid, more precisely provide experimentally accessible models of classical or quantum field theory on a curved spacetime. ${ }^{20}$

Furthermore, in these condensed matter systems the microscopic physics is well known and well behaved, so one has an effective toy model describing how the effective gravitational properties emerges from a non gravitational microscopic theory and for what could be the first deviations from standard physics due to the underlying microstructure. The realisation of an analogue black hole and the experimental study of its characteristic is the paradigm of this research line. In this parallel session we intend to have an overview of some of the most exciting developments in the area in recent years.

\section{1. "Observation of Self-Amplifying Hawking Radiation in an Analogue Black Hole Laser" by Jeff Steinhauer}

One of the most interesting developments in analogue gravity of the last years is the flourishing of experimental investigations aiming at concretely testing the predictions of the theory developed in the precedent years. Among the most interesting predictions of new effects due to the underlying microscopic structure in the analogue systems is that by Corley and Jacobson, Ref. 21, that compact ergoregions (black hole-white hole configurations, where the flow is supersonic within the two horizons) will generically show an instability if the dispersion relation is modified so that at high frequencies excitations will move faster than the low energy speed of sound (as it is the case e.g. for quasi-particle excitations over a Bose-Einstein condensate (BEC)). Interestingly, the origin of this instability has to be traced into 
the self-amplifying behaviour and interference of the two types of "negative-energy" Hawking radiation partners which get trapped in the ergoregion (hence the name of black hole laser effect) and as such it is intimately related to the Hawking effect. In Ref. 22 the effect was studied in greater detail by semi-analytical methods and applied to BEC paving the way for an experimental search.

The first talk of the parallel session by Jeff Steinhauer reported very important results in this sense as it described in great details the outcome of a series of experiments carried on at Technion, Israel, which have showed compelling evidence for this effect to actually exists in an actual BEC. After an extensive review of the theoretical case for the black-hole-laser effect, it was discussed how to create an analogue black hole in a BEC. The technique used by the Technion group consisted in accelerating the condensate by making it flow over a potential step which is furthermore moving upstream. ${ }^{23}$ This system shows this way not only an analogue of a $\mathrm{BH}$ horizon at the step but also an inner horizon corresponding to the position where the BEC flows come back to subsonic speeds after the passage of the step.

By studying the correlation patter of the associated production of quasi-particles it was shown that the expected features related to the laser instability and the associated, fainter, Hawking emission do show up in the experimental data. Furthermore, it was shown how the observed timescales and frequencies for the effect are in good agreement with the theoretical expectations given the experimental setting, lending further support to the evidence of a first detection of the laser-instability. The talk ended by discussing further investigations of the proper Hawking-emission correlation pattern which anticipated some of the results published in the subsequent article, Ref. 24, which claims the first direct observation of quantum Hawking radiation in an analogue black hole.

\section{2. "Analogue Black Holes in Quantum Fluids of Light" by Iacopo Carusotto}

The talk by Iacopo Carusotto was also devoted to the hunting for a Hawking radiation analogue albeit in a different setting: an analogue system based on a photon fluid. In one- or two-dimensional microcavity devices, photons acquire an effective mass because of spatial confinement, while an effective photon self-interaction can originate from the optical nonlinearity of the cavity medium. As a result, assemblies of many photons in the cavity can display the collective behaviour of a Bose-Einstein condensate, as well as superfluid hydrodynamic features with the low-frequency elementary excitations behaving like phonons. ${ }^{25}$

In the talk it was showed an experimental set up (see Ref. 26) consisting of such a photon fluid made to flow via a suitable laser pump hitting the apparatus at an oblique angle. The photon superfluid was in this way made to flow toward a defect so inducing a transition from subsonic to supersonic motion. The possibility to generate a black-hole analogue opens up the possibility for searching for analogue Hawking radiation. While in this setting one obtains dissipative terms that should 
be taken into account, it was shown that the usual characteristic density-density correlation pattern associated to the Hawking radiation will show up and that integration over long times might allow to clean up the signal with respect to the shot noise. Numerical simulations (see Ref. 26, 27) seem to provide good agreement with the theoretical, analogue gravity, expected results, hence suggesting a concrete chance of detection in the near future.

\section{3. "Analogue Gravity in Fluids Made by Light" by Daniele Faccio}

Photon fluids were also the subject of the talk by Daniele Faccio. However, in this case the experimental set up considered was different. It consists of a laser propagating through a defocussing non-linear medium. In this case the equations governing the space dependence of the electric field associated with the light pulse can be cast as hydrodynamical equations for a non-relativistic perfect fluid (where the time variable is associated to the space coordinate along the axis of propagation of the light). Small amplitude excitations of such a photon fluid were shown to be endowed with a Bogoliubov-type dispersion relation similar to the one appearing in standard BEC. ${ }^{28}$

Quite remarkably, the theoretical expectation for such dispersion relation was shown to be confirmed in experiments where it was accurately reconstructed for the small-amplitude noise on the laser beam. ${ }^{29}$ After a discussion of other experimental setups that can be studied in this system, it was further develop the possibility to test the gravity-analogue aspects by simulating a black-hole spacetime. This can be done both by having a constant velocity flow with varying speed of sound or by producing a gradient in the flow velocity keeping the speed of sound constant. The black hole (white hole) horizon will then corresponds to the locus of points where the flow velocity and the speed of sound equate.

The seminar then focussed on two further phenomena that can be simulated in this photon-fluid system. One is the possibility to generate vortices and analogue geometries simulating rotating black holes. These configurations could be then used to simulate well-known phenomena like superradiance. Then it was also considered the effective geometry experienced by large-amplitude density waves in the fluid. In this case one obtains a full Navier-Stokes equation for a compressible perfect fluid and consequently it leads to effective geometries with density-dependent speed of sound and background velocity flows. Interestingly, it was finally discussed the possible geometrical interpretation of shock fronts in the fluid as singularities in the acoustic metric. Also several observational results regarding incoherent shock waves in the photon fluid were presented.

\section{4. "Can One Probe the Thermal Character of Analog Hawking Radiation using Shallow Water Waves?" by Renaud Parentani}

The following talk by Renaud Parentani was dedicated to a novel analysis of a recent set of analogue gravity experiments based on shallow water waves in a flume. 
This analogue model was theoretically proposed in a seminal paper by Schutzhold and Unruh in 2002 (see Ref. 30) and later on experimentally realised for the first time in Nice by Rousseaux and collaborators. ${ }^{31}$ They observed the production of negative energy waves on a white hole horizon analogue generated by putting on the bottom of the flume a suitably smooth obstacle that locally reduces the height of the flow and hence the speed of propagation of gravity waves. It was however with the Vancouver experiment realised by Weinfurtner, Unruh and collaborators that a full claim for the detection of the classical analogue of Hawking radiation from a white hole horizon was made. ${ }^{32}$

Parentani revised the theory behind this class of experiments starting by noticing that in the Vancouver and Nice experiments, the flows were not trans-critical ( $v_{\text {flow }} / c<1$ everywhere, $c$ being the low frequency speed of the gravity waves). His analysis is based on the equations for a stationary, irrotational, two dimensional laminar flow of an inviscid incompressible fluid and for its associated linearised perturbations. The wave equation was then simplified by truncating to quartic order the "subluminal" dispersion relation which specifies that high wavenumber modes propagate slower that low wavenumber ones.

Parentani then considered the mode conversion encoding the analog Hawking effect in trans- and sub-critical flows, that is, with or without Killing horizons. When considering sub-critical flows, the analysis indicates that the emitted spectrum is very different from a Planck spectrum, albeit the ratio of the Bogoliubov coefficients associated to the positive and negative norm modes can show an approximate exponential behaviour as if a Planck spectrum was indeed emitted. The point made by Parentani was that such a ratio is a relevant diagnostic of a thermal spectrum only if the difference of the squares of the above mentioned coefficients is close to one. However, this is never the case for low frequency modes in subcritical flows because of the large transmission at low frequencies. Furthermore he showed how the scattering coefficients evolve towards their standard relativistic behaviours when increasing the maximal value of $v_{\text {flow }} / c$ from values smaller to higher than 1 . In the closing remarks, he gave a proposal for designing obstacles allowing to obtain trans-critical flows.

\section{5. "Sparsity of the Hawking Flux" by Matt Visser}

The subsequent talk, by Matt Visser, was an in depth investigation about the typical features of the Hawking flux in a black-hole evaporation process. In particular the seminar focussed on the characteristic sparsity at spatial infinity of the thermal flux related to the fact that the typical time between quanta reaching infinity is much larger than the timescale set by the energy of the quanta (by orders of magnitude).

After an historical introduction, Visser passed on to show, in a simple pureblack-body approximation of the radiation (i.e. without grey-body factors), that a sparsity indicator, i.e. the typical ratio of the frequency gap between quanta versus their typical timescale, is proportional to the ration of the square of the wavelength 
associated to the Hawking temperature divided by the area of the emitter. While this normally implies a very tiny ratio and hence a very dense distribution of thermal quanta, for a Schwarzschild-black-hole case the opposite is realised and a very sparse flux is then realised. A refinement of this treatment was shown to give basically the same figures of merit and hence to reach the same conclusions. The sparsity of the flux calls into question the validity of applying Bose-Einstein or Fermi statistics to black-hole evaporation.

The follow up of the presentation dealt with other types of black holes such as Reissner-Nördstrom, "dirty" and Kerr black holes. In all these cases the flux was shown to be even more sparse than in the Schwarzschild case. In the particular case of a Kerr black hole, it was also discussed in detail the possible role of superradiance in increasing the density of the flux albeit it was stressed the rather different nature of the two effects. A method for distinguishing the two effects was suggested and it was shown that generically the flux at infinity will be dominated by superradiance until the black hole is sufficiently spin down so that the standard Hawking effect could take over.

Based on the sparsity of the flux, the final part of the talk advanced a reinterpretation of Hawking evaporation as a 3-body-cascade process (initial black hole $\rightarrow$ final black hole + Hawking radiation). The relativistic kinematics of this process was analysed, for massless quanta and a Schwarzschild black hole, showing that the typical energy of the emitted quanta scales as the ratio between the difference of the squared rest masses of the initial and final black hole states, and twice the initial rest mass of the black hole. It was suggested that this law should be related to the first law of black hole mechanics and that extension of the analysis to more general setting would be necessary.

\section{6. "Black Hole-Moving Mirror I: Correspondence" by Paul Anderson}

The session moved on with a talk by Paul Anderson, about the black hole-moving mirror correspondence. This correspondence is text-book material by now having been explored in the late seventies by several authors, most noticeably by Davies and Fulling. Basically, it can be shown that the derivation of the Hawking radiation as the late time emission of a stellar collapse leading to a black hole can be reduced to the study of an exponentially receding mirror in flat spacetime. As such most studies have focussed on such late-time radiation and its thermal character and rarely to the early-times behaviour where in general one does not expect any correspondence between the mirror and the black-hole emission.

Anderson's presentation focussed on the correspondence between the black hole and mirror phenomenology searching for a possible situation case in which radiation over the entire history of a black hole and moving mirror is the same. The starting point was the analysis of a $(1+1)$ dimensional collapsing null shell and a massless, minimally coupled, scalar field. By adopting standard techniques of quantisation 
on such a spacetime and matching the affine coordinates on past and future null infinities the Bogolyubov coefficients describing the associated particle production were computed.

It was then considered a moving-mirror model and showed that for a specific trajectory (including the so called Lambert or Product-Log function) one can find exactly the same Bogoliubov coefficients describing the black-hole case. This is hence an example where the black hole-mirror correspondence is exact. Finally it was discussed that the results extend to the case of $3+1$ dimensions if grey-body factors are neglected and scattering effects ignored.

\section{7. "Black Hole-Moving Mirror II: Particle Creation" by Michael Good}

The next talk by Michael Good was intended as a follow up of the previous one. Indeed, also in this talk the black hole-mirror correspondence was explored. In particular, Good investigated the time dependence of the particle production process and the approach to a thermal distribution at late times. Again, it was considered a $(1+1)$ dimensional massless, minimally coupled, scalar field and for the moving mirror it was taken the specific trajectory discussed in the previous talk which mimic a black-hole formation by a collapsing null shell (even at early times).

Contrasting this case with the simple case of a mirror trajectory generating an eternal thermal flux (and hence mimicking the Unruh effect rather than the Hawking one), it was computed the time dependence of the energy flux for the field in the mirror spacetime. It was shown that while the late-time flux approaches thermally and the standard features of the steady Hawking flux, the early-time behaviour is time dependent and monotonic. In this sense, it was stressed how moving mirrors provide a simple laboratory to construct the mathematical machinery needed to understand the time evolution of black hole evaporation.

\section{8. "The Role of Causality in Tunable Fermi Gas Condensates: From Creation of Vortices to Analog Model of FRW Universes" by Da-Shin Lee}

The first of the last two talk of the session was dedicated to analogue gravity systems based on tunable Fermi gases with Feshbach resonances (i.e. in a setting were the scattering length can be effectively controlled). These systems are characterised by two phases: for negative scattering length (attractive interaction) there can be a weak fermion pairing leading to a BCS (Bardeen, Cooper, Schrieffer) theory of Cooper pairs (superconductor phase), while for positive scattering length (repulsive interaction) a strong fermionic pairing is induced leading to a BEC theory of diatomic molecules. At the BCS/BEC crossover the Feshbach resonance implies a divergence and sign reversal of the scattering length.

The studies reported in this talk were all based on a non-relativistic system describing a condensate of fermionic atoms and molecular bosons (two fermions bound 
states), the features considered were the spontaneous vortex production in fast-field quenches, the spontaneous particle production in fast quenches and sound-cone fluctuations. The outcome of the reported investigations is that vortex production should be expected in fast quenches but analogue particle production in this setting would be hard to observe. Detection of phonons are instead more promising.

Indeed, it was discussed an analogue model of cosmological particle production by considering the superfluid phase in a fermonic condensate (both in BCS and BEC phases). Using standard techniques (see e.g. Ref. 20) it was shown that the timedependent spacetime (via the possibility to tune the scattering length) could mimic a cosmological particle production although the Lorentz breaking dispersion relation of the quasiparticles would lead to new phenomenology e.g. w.r.t. the standard inflationary scenarios.

Finally it was discussed a model of sound cone fluctuations which would simulate a conjectured similar effect on light cones due to quantum gravity. This effect could be tested via the study of the variation in travel time of the sound waves between a source and a detector. The analysis presented seems to imply that a possible observation in the lab of such analogue quantum-gravity phenomenology could be viable.

\section{9. "High Overtone Quasinormal Modes of Analog Black Holes and the Small Scale Structure of the Background Fluid" by Ramin Daghigh}

The last talk of the session was dedicated to an analogue gravity model investigating the properties of quasi-normal modes (QNMs) in analogue black holes. QNMs are damped harmonic oscillations of the background geometry that in the case of blackhole geometries allow to reconstruct many of the spacetime features. After reviewing QNMs and their mathematical theory, the seminar focused on highly-damped modes (those with short relaxation times). In fact, there is some evidence (relieved in the talk) that these are those which might probe the possible structure of spacetime at short scales.

It was then argued that a possible probe of this conjecture would be to look for an analogue of this phenomenology in an analogue system where a microscopic description of the background geometry is known. Indeed, the second part of the talk focussed on discussing QNM (not only the least damped modes but the higher modes are useful to identify the corresponding classical geometry) in analogue black holes generated in BEC systems. The fact that quasiparticles in these systems show a modified, Bogolyubov, dispersion relations suggests that high-overtone QNMs will in this case be determined by the so called BEC quantum potential, which is linked to the microscopic structure of the background. However, a direct exploration of an analogue $1+1$ geometry leads to results rather different from the GR ones. QNM are present (while in GR in $1+1$ they are absent) and with a continuous spectrum (in GR in $3+1$ they are instead discrete). Finally, no overtones are found. This 
seems to suggest that the $1+1$ model is too simple to encode interesting features and as such a higher dimensional analysis would be needed.

\section{Conclusions}

The BH6 parallel session of this Marcel Grossmann meeting was dedicated to two booming fields of research on black hole physics. Regular-black-hole models seem a natural possibility once one realises that the singularities of classical GR should be tamed by quantum gravity effects. The absence of a full fledged event horizon seems to remove many problematic features of the standard picture while still allowing for many of the tantalising quantum properties of black holes. It is still unclear whether such proposals would be viable for solving the information loss problem, but at least the parallel session talks showed clearly that these models have common features that imply a distinctive phenomenology. These features suggest specific signatures possibly relevant for astrophysics and as such amenable to observational tests in the near future.

On the analogue gravity side, the session represented many of the most active trends in the field. In particular, it has to be stressed that many talks dealt with experiments and in some cases showed the possibility to test some features of quantum field theory in curved space-times that were till few years ago restricted the theoretical realm. In this case we think that the parallel session gave a vivid picture of the bustling activity in the field and showed some of its future potential developments.

In conclusion, we had a very lively parallel session with a very broad range of topics. We look forward to the next session on these topics in MG15!

\section{Acknowledgments}

Financial support for C.B. was provided by the Spanish Ministry through the projects FIS2011-30145-C03-01 and FIS2014-54800-C2-1 (with FEDER contribution), and by the Junta de Andalucía through the project FQM219. S.L. wish to acknowledge the John Templeton Foundation for the supporting grant \#51876.

\section{References}

1. A. D. Sakharov, Sov. J. Exp. Theor. Phys. 22, 241 (1966).

2. E. B. Gliner, Sov. J. Exp. Theor. Phys. 22, 378 (1966).

3. R. Penrose, Phys. Rev. Lett. 14, 57-59 (1965).

4. J. Bardeen, Proc. Int. Conf. GR5, Tbilisi, p. 174 (1968).

5. A. Borde, Phys. Rev. D 50, 3692-3702 (1994). [arXiv:gr-qc/9403049].

6. A. Borde, Phys. Rev. D 55, 7615-7617 (1997). [arXiv:gr-qc/9612057].

7. I. Dymnikova, Gen. Rel. Grav. 24, 235-242 (1992).

M. Mars, M. M. Martín-Prats and J. M. M. Senovilla, Class. Quantum Grav. 13, L51 (1996). 
E. Ayon-Beato and A. Garcia, Phys. Rev. Lett. 80, 5056-5059 (1998) [arXiv:gr-qc/9911046].

C. Barceló, R. Carballo-Rubio and L. J. Garay, Int. J. Mod. Phys. D 23, no. 12, 1442022 (2014). [arXiv:1407.1391 [gr-qc]].

K. A. Bronnikov, Phys. Rev. D 63, 044005 (2001). [arXiv:gr-qc/0006014].

K. A. Bronnikov, Phys. Rev. D 64, 064013 (2001). [arXiv:gr-qc/0104092].

S. A. Hayward, Phys. Rev. Lett. 96, 031103 (2006). [arXiv:gr-qc/0506126].

G. J. Olmo, D. Rubiera-Garcia and H. Sanchis-Alepuz, European Phys. J. C 74, 2804 (2014). [arXiv:hep-th/1311.0815].

G. J. Olmo and D. Rubiera-Garcia, Nonsingular Black Holes in $f(R)$ Theories, ArXiv e-prints (2015), [arXiv:hep-th/1509.02430].

8. A. Ashtekar and M. Bojowald, Class. Quantum Grav. 23, 391-411 (2006). [arXiv:gr-qc/0509075].

A. Ashtekar, V. Taveras and M. Varadarajan, Phys. Rev. Lett. 100, 211302 (2008). [arXiv:gr-qc/0801.1811].

S. Hossenfelder and L. Smolin, Phys. Rev. D 81, 064009 (2010). [arXiv:gr-qc/0901.3156].

H. Kawai, Y. Matsuo and Y. Yokokura, Int. J. Mod. Phys. A 28, 1350050 (2013). [arXiv:hep-th/1302.4733].

H. Kawai and Y. Yokokura, Int. J. Mod. Phys. A 30, 50091 (2015). [arXiv:1409.5784].

R. Torres, Phys. Lett. B 733, 21-24 (2014). [arXiv:gr-qc/1404.7655].

V. .P. Frolov, 18th International Seminar on High Energy Physics (Quarks 2014) Suzdal, Russia, June 2-8, (2014) [arXiv:hep-th/1411.6981].

M. Bojowald, Front. Phys. 3, 33 (2015). [arXiv:gr-qc/1409.3157].

R. Gambini, J. Olmedo and J. Pullin, Class. Quantum Grav. 31, 095009 (2014). [arXiv:gr-qc/1310.5996].

R. Torres and F. Fayos, Phys. Lett. B 747, 245-250 (2015). [arXiv:gr-qc/1503.07407].

C. Rovelli and F. Vidotto, Int. J. Mod. Phys. D 23, 42026 (2014). [arXiv:gr-qc/1401.6562].

A. Barrau, A. and C. Rovelli, Phys. Lett. B 739, 405 (2014). [arXiv:gr-qc/1404.5821].

C. Bambi, D. Malafarina and L. Modesto, Phys. Rev. D 88, 044009 (2013). [arXiv:gr-qc/1305.4790].

Y. Liu, D. Malafarina, L. Modesto and C. Bambi, Phys. Rev. D 90, 044040 (2014). [arXiv:gr-qc/1405.7249].

Y. Zhang, Y. Zhu, L. Modesto and C. Bambi, European Phys. J. C 75, 96 (2015). [arXiv:gr-qc/1404.4770].

S. K. Modak, L. Ortíz, I. Peña and D. Sudarsky, Phys. Rev. D 91, 124009 (2015). [arXiv:gr-qc/1408.3062].

S. K. Modak, L. Ortíz, I. Peña, D. Sudarsky, Gen. Rel. Grav. 47, 120 (2015). [arXiv:gr-qc/1406.4898].

S. W. Hawking, ArXiv e-prints (2015), [arXiv:hep-th/1509.01147].

G. t'Hooft, Diagonalizing the Black Hole Information Retrieval Process, ArXiv eprints (2015), [arXiv:gr-qc/1509.01695].

V. P. Frolov and G. A. Vilkovisky, Grossmann Mtg.1979:0455, p. 0455 (1979).

V. P. Frolov and G. A. Vilkovisky, Phys. Lett. B 106, 307-313 (1981).

T. Roman and P. Bergmann, Phys. Rev. D 28, 1265-1277 (1983).

A. Ashtekar and M. Bojowald, Class. Quantum Grav. 22, 3349-3362 (2005). [arXiv:gr-qc/0504029]. 
C. Bambi, D. Malafarina and L. Modesto, Eur. Phys. J. C 74, 2767 (2014). [arXiv:gr-qc/1306.1668].

J. M. Bardeen, ArXiv e-prints (2014), [arXiv:gr-qc/1406.4098].

H. Kawai and Y. Yokokura, Interior of Black Holes and Information Recovery, ArXiv e-prints (2015), [arXiv:hep-th/1509.08472].

9. V. P. Frolov and G. A. Vilkovisky, Grossmann Mtg.1979:0455, p. 0455 (1979).

10. V. P. Frolov and G. A. Vilkovisky, Phys. Lett. B 106, 307-313 (1981).

11. C. R. Stephens, G. 't Hooft and B. F. Whiting Class. Quantum Grav. 11, 621 (1994). [gr-qc/9310006].

12. P. Hajicek and C. Kiefer, Int. J. Mod. Phys. D 10, 775 (2001). [gr-qc/0107102].

13. A. Ashtekar and M. Bojowald, Class. Quantum Grav. 22, 3349-3362 (2005). [arXiv:gr-qc/0504029].

14. G. 't Hooft, Nucl. Phys. Proc. Suppl. 203-204, 155 (2010). link.

15. R. Gambini and J. Pullin, PoS FFP 14, 038 (2015). [arXiv:1408.3050 [gr-qc]].

16. R. Gambini and J. Pullin, Class. Quantum Grav. 32, no. 3, 035003 (2015). [arXiv:1408.4635 [gr-qc]]

17. C. Barceló, R. Carballo-Rubio, L. J. Garay and G. Jannes, Class. Quantum Grav. 32, no. 3, 035012 (2015). [arXiv:1409.1501 [gr-qc]].

18. H. M. Haggard and C. Rovelli, Phys. Rev. D 92, no. 10, 104020 (2015). [arXiv:1407.0989 [gr-qc]].

19. M. Ambrus and P. Hajicek, Phys. Rev. D 72, 064025 (2015). [gr-qc/0507017].

20. C. Barceló, S. Liberati and M. Visser, Living Rev. Rel. 8, 12 (2005) [Living Rev. Rel. 14, 3 (2011)] [gr-qc/0505065].

21. S. Corley and T. A. Jacobson, Phys. Rev. D 59, 1-12, 124011 (1999).

22. S. Finazzi and R. Parentani, New J. Phys. 12, 095015 (2010). [arXiv:1005.4024 [condmat.quant-gas]].

23. J. Steinhauer, Nature Phys. 10, 864 (2014). [arXiv:1409.6550 [cond-mat.quant-gas]].

24. J. Steinhauer "Observation of thermal Hawking radiation and its entanglement in an analogue black hole," ArXiv e-prints (2015), [arXiv:gr-qc/1510.00621].

25. I. Carusotto and C. Ciuti, Rev. Mod. Phys. 85, 299 (2013).

26. H. S. Nguyen, D. Gerace, I. Carusotto, D. Sanvitto, E. Galopin, A. Lematre, I. Sagnes, J. Bloch, and A. Amo, Phys. Rev. Lett. 114, 036402 (2015).

27. D. Gerace, I. Carusotto, Phys. Rev. B 86, 144505 (2012).

28. I. Carusotto, Proc. R. Soc. A 470, 20140320 (2014).

29. D. Vocke, T. Roger, F. Marino, E. M. Wright, I. Carusotto, M. Clerici, and D. Faccio, Optica 2, 484 (2015).

30. R. Schützhold and W. G. Unruh, Phys. Rev. D 66, 1-13, 044019 (2002).

31. G. Rousseaux, C. Mathis, P. Maissa, T. G. Philbin and U. Leonhardt, New J. Phys. 10, 053015 (2008).

32. S. Weinfurtner, E. W. Tedford, M. C. J. Penrice, W. G. Unruh and G. A. Lawrence, Phys. Rev. Lett. 106, 021302 (2011). [arXiv:gr-qc/1008.1911]. 